\title{
Vibration Diagnosis of Failure of Mechanical Coupling between Motor and Pump Rotors
}

\author{
Jyoti K. Sinha and A. Rama Rao \\ Vibration Laboratory, Reactor Engineering Division, Bhabha Atomic Research Centre, Mumbai 400 085, India
}

(Received 10 August 2004; accepted 3 May 2005)

\begin{abstract}
A failure of the mechanical coupling between the rotors of the pump and motor for two pump assemblies was observed since the pumps commissioned in 1984. The use of vibration based conventional condition monitoring during the pump operation could not identify the root cause, though high levels of vibration were observed. Modal tests conducted on the complete assembly of pumps and piping layout identified resonance as the root cause. Vibration measurements, data analysis, and observations that led to the identification of the root cause for the failure of the mechanical coupling have been reported here as a case study.
\end{abstract}

\section{INTRODUCTION}

A $100 \mathrm{MW}$ research reactor in India has two overhead storage tank (OHST) makeup pumps, which are part of the emergency core cooling system. These pumps are of the centrifugal type, and high levels of vibration were observed in the pump assemblies since the pumps commissioned in 1984 . Recently, a failure of the mechanical coupling between the pump and the driving motor shafts was reported for both of the pumps. Mechanical maintenance personnel noticed the development of a crack in the couplings during a routine inspection, but visual examination revealed no affect on the rotors. The pumps were put back into operation after repair and replacement of the defective parts. The rotors were adequately aligned, and the motor was balanced before putting it back into operation. The use of conventional vibration based condition monitoring (based on the appearance of different frequency components related to pump RPM) ${ }^{1,2}$ during pump operation was not enough to provide significant insight into the malfunctioning of the pumps so that the problem could be rectified permanently. Hence, modal tests were conducted on the pump assemblies and the piping layout when both the pumps were not in operation in order to understand the dynamic behaviour of the entire assembly of pumps and connected piping. Modal tests were also conducted when Pump 1 was operational. In addition to the modal tests, vibration measurements were also carried out during the normal operation of Pump 1. Vibration measurements and data analyses that led to identification of the root cause for the failure of the mechanical coupling have been discussed here as a case study.

\section{DESCRIPTION OF PUMP ASSEMBLY AND PIPING LAYOUT}

Figure 1 gives the schematic of the pump assembly. It is a horizontally mounted centrifugal pump with an axial inlet and a radial outlet. The pump is driven by an electric motor $(60 \mathrm{~kW})$ operating at $3000 \mathrm{RPM}$. The complete assembly of the pump is mounted on a base plate, which directly rests on a rigid concrete floor. The schematic of both the pumps and the piping layout is shown in Fig. 2. The ends 1 to 4 of the inlet piping, shown in Fig. 2, pass through concrete walls of the pump house and are expected to be clamped there. The end 5 of the inlet piping is a closed end which rests on a vertical column that supports the weight of the pipe.

\section{MODAL TESTS AND VIBRATION MEASUREMENT}

Modal testing ${ }^{3}$ is an experimental means of characterising the dynamic properties (natural frequency, damping, and mode shape) of structures and machines. Modal tests were conducted on both the pumps and their piping when both pumps were not operating, as well as when Pump 1 was operational. The impulse-response method was used for the modal tests. ${ }^{3}$ An instrumented hammer was used to give impulse excitation to the pump assembly up to a frequency band of around $500 \mathrm{~Hz}$, and the responses of the assembly from different locations in different directions $(x-$ along shaft axis, $y$ - vertical, $z$ - lateral to shaft axis) were collected using a number of accelerometers. Since a 16 channel digital data recorder was available for storing measured data at the site, a total of 15 accelerometers (B\&K 4396) were used for responses and one channel for force. The modal tests on the entire pump assemblies and piping together were completed in two measurement sets. Figure 3 shows the schematic of the measurement locations used in the modal tests through a "wire frame" diagram of the base structure, pump casing, and inlet piping for both the sets. In addition to the modal tests, the vibration measurements were also carried out during the operation of Pump 1.

\section{DATA ANALYSIS AND RESULTS}

The measured vibration responses during one pump operation and the impulse-response data of the modal tests were analysed. The overall (peak to peak) displacement and the rms velocity at the Pump 1 bearing in both vertical and horizontal directions were found to be $(58,54)$ micron and $(7.96$, $7.75) \mathrm{mm} / \mathrm{s}$ respectively, which are much above the acceptable limits as per the Hydraulic Institute ${ }^{4}$ and the ISO code ${ }^{5}$. Initially, the impulse-response test data during the operation of Pump 1 was analysed. A few typical time signals and the frequency response functions (FRFs) are shown in Figs. 4 and 5. It was difficult to identify the modes accurately because 